\title{
Components of working memory and somatic markers in decision making
}

\author{
TINA L. JAMESON, JOHN M. HINSON, and PAUL WHITNEY \\ Washington State University, Pullman, Washington
}

\begin{abstract}
According to Damasio's somatic marker hypothesis, affective reactions ordinarily guide and simplify decision making. In an earlier study, we used a modified version of the gambling task developed by Bechara and colleagues so that we could explore the relations among decision making, working memory (WM) load, and formation of somatic markers. This prior work found that an increased WM load produced by secondary tasks interfered with the development of somatic markers and led to poorer gambling task performance. In the present study, we tested whether secondary tasks affect the executive functions of WM, verbal buffering, or both. Our findings indicate that verbal buffering alone does not interfere with gambling task performance or the development of somatic markers. Interference with the executive functions of WM is necessary to disrupt gambling performance and somatic markers.
\end{abstract}

Damasio and colleagues have proposed the somatic marker hypothesis, which argues that affective states associated with prior decision outcomes are used to guide future decisions (Bechara, H. Damasio, \& A. R. Damasio, 2000; Damasio, 1994). According to this hypothesis, good decisions result, in part, because people feel that one course of action will be bad, and that directs them toward other options. The anticipatory reaction, a somatic marker, allows potentially bad choices to be anticipated and, presumably, makes complex decision making simpler and more efficient. A number of studies in which the somatic marker hypothesis has been explored have used the gambling task (GT), a laboratory decision-making task involving hypothetical gambles (see Bechara, A. R. Damasio, H. Damasio, \& Anderson, 1994).

The somatic marker hypothesis was originally designed to explain the peculiar decision-making problems of people with injuries to the ventromedial prefrontal cortex (VMPFC; Damasio, 1998). Most patients with damage to the VMPFC retain the general intellectual ability needed to make good decisions, yet they typically make poor decisions that often lead to financial ruin and serious difficulties in normal social life. Patients with VMPFC injury continue to have emotional reactions to good and bad consequences of their actions. But what VMPFC patients lack is the anticipation of the bad consequences of action that would ordinarily steer them away from poor choices (Bechara, Tranel, Damasio, \& Damasio, 1996). This lack of anticipation for the consequences of actions in daily life has been called "myopia for the future" (e.g., Bechara, Dolan, \& Hindes, 2002).

Correspondence concerning this article should be addressed to J. M. Hinson, Department of Psychology, Washington State University, Pullman, WA 99164-4820 (e-mail: hinson@mail.wsu.edu).
An intriguing claim of the somatic marker hypothesis is that the affective responses that guide decision making are a source of influence separate from the purely cognitive factors that depend on the functions of working memory (WM). WM is that part of the cognitive system used to hold a limited amount of information in the focus of attention (Smith \& Jonides, 1999). WM processes are one of the most important functions of the frontal cortex (e.g., Fuster, 1999; Miller \& Cohen, 2001; Shallice \& Burgess, 1991). Damasio and colleagues have argued that ventral regions of the PFC support decision making based on somatic markers, whereas dorsal regions of PFC support WM function (Bechara et al., 2000). However, the relation between somatic markers and WM, and their implementation in the frontal cortex, remains unclear (see Krawczyk, 2002). Whereas VMPFC patients can show normal performance on delayed response tasks, indicative of a normally functioning WM (Bechara, H. Damasio, Tranel, \& A. R. Damasio, 1998), patients with damage to more posterior frontal regions have both decision-making problems and WM deficits (Bechara et al., 1998). Furthermore, recent evidence indicates that ventral and dorsal regions of the frontal cortex interact in decision making (Manes et al., 2002).

In prior work with a normal population, we found that GT performance and the establishment of somatic markers is dependent on available WM capacity (Hinson, Jameson, \& Whitney, 2002). We used a version of the GT that was more challenging than the original and added secondary tasks designed to load WM. The secondary tasks included a digit string to be retained during each gambling trial and random number generation. As compared with a no WM load control condition, participants made poorer GT choices in the WM load conditions. In addition, anticipatory skin conductance response (SCR) amplitude, an index of somatic markers, did not appear in WM load con- 
ditions associated with poor GT performance. Thus, by interfering with WM function, we prevented the development of the affective reactions that appear to guide good GT decision making.

The data obtained by Hinson et al. (2002) demonstrated that performance on the GT and formation of a somatic marker are dependent on WM. However, WM is a multicomponent system (e.g., Baddeley, Chincotta, \& Adlam, 2001), and more data are needed to specify what aspects of WM are involved in the GT. Consider the finding that GT performance declines with a concurrent digit load, as compared with a no-load control condition. Such digit loads have two potential sources of influence: (1) They consume central executive resources, leaving less processing capacity available for the primary task, and (2) they interfere with verbal short-term storage by occupying the phonological loop (Baddeley et al., 2001; Baddeley \& Hitch, 1974). Therefore, our previous data could reflect the need for central executive resources in GT performance, the use of the phonological loop for maintaining verbal codes during the GT, or a combination of both factors.

The central executive interpretation of our results suggests that WM resources are needed to maintain at least a rough running average for each deck and that the running average influences the establishment of a stable affective response to each deck. Extrinsic WM loads (digit maintenance or random number generation) consume some of the resources needed for keeping track of outcomes, so performance suffers under load conditions. It is also possible that load effects on GT can be explained, instead, by interference with the phonological loop. Recent data indicate a contribution of the phonological loop to complex task performance (Baddeley et al., 2001; Dunbar \& Sussman, 1995; Miyake \& Shah, 1999). Baddeley et al. showed that articulatory suppression, induced by repeating a welllearned sequence such as $1-2-3-1-2-3$, can interfere with the control of actions even though such suppression does not consume central executive resources. The reason that articulatory suppression can influence performance without great demands on attention is that it blocks the use of the phonological loop, which is sometimes used to hold a verbal "program" for controlling actions. Thus, if a verbally mediated strategy is used to perform the GT, our prior results may reflect load effects on the phonological loop. Either maintaining a concurrent digit load or keeping track of randomly generated digits could interfere with the use of the phonological loop.

In the present study, we replicated the key conditions of Hinson et al.'s (2002) study and also included an articulatory suppression condition, in order to determine whether all or part of the effects of load manipulations on GT performance are due to the disruption of verbal strategies that require the use of the phonological loop.

\section{METHOD}

\section{Participants}

The participants were 20 graduate and undergraduate students at Washington State University, who were paid for their participation.
The participants were between the ages of 20 and 28 years, and $60 \%$ were female.

\section{Procedure}

The participants were given a brief oral description of the task, paraphrased as follows:

\begin{abstract}
In this experiment, you will be asked to make hypothetical gambles similar to those made in a card game. You will start with a fixed sum of money, and the computer will prompt you to make repeated choices. For each choice, you will sometimes lose money and sometimes win money. Your task is to discover the best way to make choices, so that at the end of the session you will have the highest amount of money possible. You will also be asked to perform some additional cognitive tasks while you are making your choices. In one block of trials, a string of five numbers will be presented to be remembered, such as "Remember 25341." Rehearse the five numbers so that you will be able to retain them during the gambling trial. After your gambling choice, you will be prompted to respond, such as "The number to the right of 5." In this case, the correct response is "3." Make the response " 3 " on the numeric keypad. In another block of trials, when given an on-screen prompt, we will ask you to continually repeat the word "the." Please continue to say "the" until you have made a gambling choice. In another block of trials, after each gambling choice is made, a number will be displayed, and you will be asked to make that response on the numeric keypad.
\end{abstract}

The participants were seated in front of individual computer terminals and were told to begin when ready. A program written in Microsoft Basic provided written instructions and practice trials to illustrate the procedure and collected all the data from the experiment. The participants could practice as much as they wished, although most people took only a few practice trials to familiarize themselves with the procedure.

Each participant was given three blocks of 100 trials for each secondary task. The participants were randomly assigned to one of the six possible orders of secondary task, so that we had roughly equal numbers for each order. On each gambling trial, the computer gave a choice among four options. The options were displayed as four rectangles, about the area of a playing card, colored red, blue, green, or brown with the number 1, 2, 3, or 4 in the middle. Outcomes for choices were based on random selection among a set of gains and losses. One choice, the good option, produced small gains with even smaller losses over repeated choices. Another choice, the neutral option, resulted in neither gain nor loss over repeated choices. The other two choices, the badl and bad 2 options, produced large gains with even larger losses, over repeated choices. Table 1 provides a summary of the choice outcomes arranged for the four options.

The positions of the four options were randomly selected but remained consistent for the 100 gambling trials of the secondary task. The current tally of money, $\$ 2,000$ at the beginning of each secondary task, was displayed below the options. On each trial, once a choice had been made on the numeric keypad, the color of the option was replaced by white lettering on a black background, which stated "You won \$X" or "You lost \$X," where X was the amount gained or lost on that trial. The tally immediately changed, and there was a pause of about $1.5 \mathrm{sec}$ for the participant to note the results of the choice.

In the digit maintenance condition, before each gambling choice, the participants were given a string of five digits, composed of the numbers $1-5$ randomly arranged, which was presented for $2 \mathrm{sec}$. The

Table 1

Payoff Parameters for Choices in Experiment 1

\begin{tabular}{lrrrrc}
\hline Choice Type & \multicolumn{1}{c}{ Min } & Max & \multicolumn{1}{c}{$M$} & $S D$ & $P$ (Loss) \\
\hline Good & -75 & 50 & 20 & 95 & .1 \\
Neutral & -75 & 50 & 0 & 54 & .5 \\
Bad1 & -350 & 100 & -35 & 158 & .5 \\
Bad2 & $-1,250$ & 100 & -35 & 427 & .1 \\
\hline
\end{tabular}


participants were asked to retain the digit string until they had made the gambling choice. After making a gambling choice, the participants were asked to identify the digit to the right of a randomly selected digit in Positions 1-4. In the articulatory suppression condition, the participants were prompted to begin saying "the" for $2 \mathrm{sec}$ prior to the gambling choice and to continue until a choice had been made. In the keypad response condition, after a gambling choice had been made, a number from 1 to 9 was displayed, and the participant was asked to press the corresponding number on the numeric keypad.

\section{Skin Conductance Response}

SCR was recorded by means of a Contact Precision Instruments SC5 SA skin conductance monitor. Conducting electrodes about $1 \mathrm{~cm}$ in diameter were attached to the left hand on the interior of the medial phalanx of the index finger and the middle finger. A drop of conductivity gel was applied between the skin and each of the two electrodes to ensure consistent ohmic contact. The electrodes were secured to the fingers by specialized double adhesive tape rings. Every $0.05 \mathrm{sec}$, the computer sampled a serial port that provided continuous skin conductance level (SCL) in microsiemens. Each SCL sample and a real-time marker were retained for later analysis.

For analysis of affective responsiveness, we measured changes in anticipatory SCR amplitude (e.g., Dawson, Schell, \& Filion, 2000; Hinson, Jameson, \& Whitney, 2002). At the beginning of each trial, SCL was sampled to establish the baseline level for that trial. The baseline SCL value was the mean for SCL samples taken during the beginning $0.5 \mathrm{sec}$ of the trial. SCL was then continuously sampled until a gambling choice had been made, to determine peak SCL. The SCR amplitude for the trial was calculated as the difference between peak SCL and baseline SCL on that trial. Any difference between peak SCL and baseline SCL of less than 0.01 microsiemen was considered to be no response on that trial. This SCR amplitude was measured prior to the gambling choice.

The specific timing of the SCR amplitude measure depended on the secondary task and the latency of each participant's choices during the primary gambling task and the secondary WM tasks. The se- quence of trial events in each secondary task condition was as follows: (1) keypad task, 1.5-sec delay-2-sec wait-gambling choice1.5 -sec display tally-keypad response; (2) articulatory suppression task, 1.5-sec delay-2-sec "the" prompt-gambling choice-1.5-sec display tally- $0.5 \mathrm{sec}$ delay; and (3) digit maintenance task, 1.5 -sec delay-2-sec digit string-gambling choice-1.5-sec display tallyrecall digit.

\section{RESULTS AND DISCUSSION}

Figure 1 shows the proportion of good GT choices made during each secondary task across four blocks of 25 trials. More good choices were made during the keypad and articulatory suppression tasks than during the digit maintenance task. Moreover, performance improved across blocks for keypad and articulatory suppression, but not for digit maintenance. A 3 (secondary task) $\times 4$ (trial block) repeated measures analysis of variance (ANOVA) of good choices, with significance set at $p<.05$, confirms these observations. There was a significant effect of secondary task $[F(2,38)=7.406]$ and an interaction of task and trial block $[F(6,114)=2.492]$. Simple contrasts within each trial block revealed fewer good GT choices for the digit maintenance task during Blocks 3 and 4, as compared with either the articulatory suppression $[F(1,19)=9.833$; $F(1,19)=6.915]$ or the keypad $[F(1,19)=10.719$; $F(1,19)=8.942]$ secondary tasks. The proportions of good choices between the articulatory suppression and the keypad tasks did not differ $(F<1)$. Thus, the articulatory suppression task, designed to interfere with the buffering of phonological information, resulted in GT performance comparable to that with the keypad task, which did not load WM.

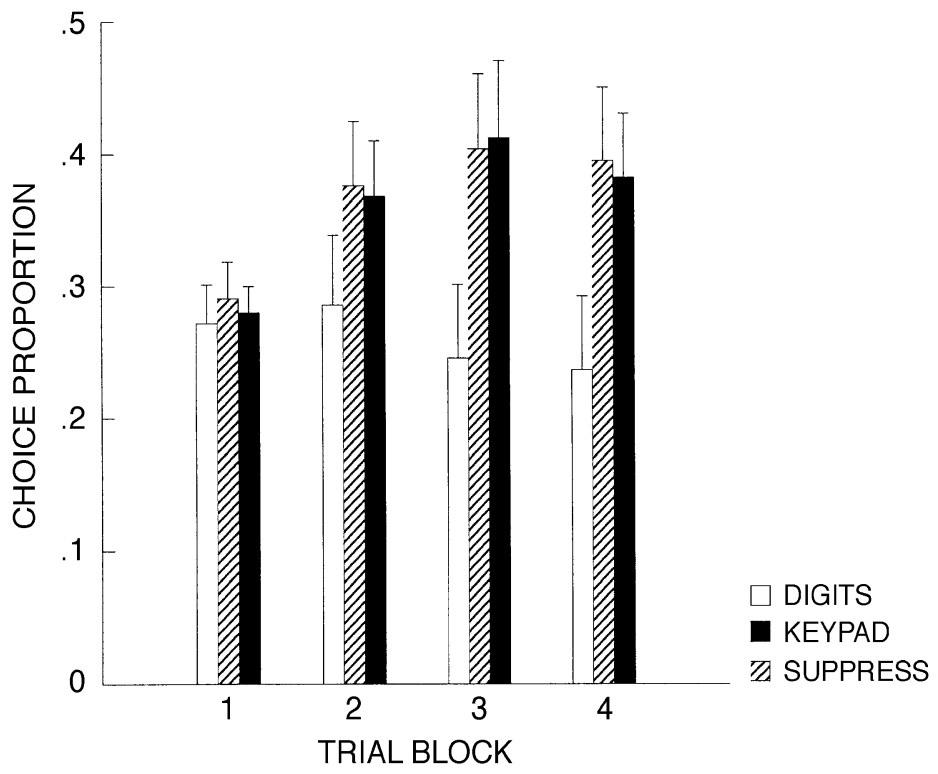

Figure 1. Mean good gambling task choices during four successive blocks of 25 trials for digit maintenance (unfilled), articulatory suppression (hatched), and keypad (filled) secondary task conditions. Error bars are the standard errors of the mean. 
Figure 2 shows anticipatory SCR amplitude for each type of GT choice - good, neutral, and bad (combining bad 1 and bad2) - during the three secondary tasks, across 25 -trial blocks. There is no consistent difference in SCR amplitude among these choices in any of the four blocks of trials for digit maintenance, shown in the left panel. A 3 (choice type) $\times 4$ (trial block) repeated measures ANOVA of anticipatory SCR amplitude indicated no reliable differences $(F<1)$.

A different pattern is observed in the middle panel of Figure 2, which shows anticipatory SCR amplitude for GT choices during the articulatory suppression task. By the third block of trials, SCR amplitude to neutral and bad choices was greater than SCR amplitude to good choices. A 3 (choice type) $\times 4$ (trial block) repeated measures ANOVA confirmed significant effects of trial block $[F(3,57)=5.882]$ and type $[F(2,38)=4.764]$ and a significant trial block $\times$ type interaction $[F(6,114)=2.317]$. Simple contrasts for each trial block show that SCR amplitude for good choices was smaller than that for neutral and $b a d$ choices in Block $3[F(1,19)=4.435 ; F(1,19)=$ $4.559]$ and Block $4[F(1,19)=8.742 ; F(1,19)=7.953]$, with no consistent differences in SCR for neutral and bad choices $(F<1)$.

The pattern of results for the keypad task (right panel of Figure 2) is similar to the pattern for articulatory suppression. A repeated measures ANOVA showed a significant effect of type $[F(2,38)=5.26]$ and a significant trial block $\times$ type interaction $[F(6,114)=2.323]$ but no significant effect of block $(F<1)$. Simple contrasts for each trial block show that SCR amplitude for good choices was smaller than that for neutral and bad choices in Block $3[F(1,19)=5.955 ; F(1,19)=10.405]$ and Block 4
$[F(1,19)=4.897 ; F(1,19)=5.553]$, with no consistent differences in SCR for neutral and bad choices $(F<1)$.

Anticipatory SCR amplitude reflected affective responses occurring after the trial began but before the gambling choice was made. It is possible that the reaction to outcomes of choices could be confounded with the anticipation of outcomes. To ensure that no such confound existed, we examined SCR amplitude as a function of prior choice in a 3 (choice type) $\times 4$ (trial block) repeated measures ANOVA. We also examined SCR amplitude as a function of prior choice outcome - that is, a gain or lossand performed a 2 (outcome type) $\times 4$ (trial block) repeated measures ANOVA. There were no significant effects in these analyses. Accordingly, the SCR amplitude differences we report reflect the anticipation of choice outcomes.

Consistent with our earlier findings (Hinson et al., 2002), a somatic marker was indicated by a consistent difference in anticipatory SCR between the better and the worse choices. The best GT performance occurred in those conditions in which there was a clear difference among choices in anticipatory SCR amplitude, such as the keypad and the articulatory suppression tasks. When there was no differentiation among anticipatory SCR amplitude for choices, as in the digit maintenance task, there was correspondingly poorer GT performance. Thus, a relatively strong and consistent affective reaction in anticipation of inferior choices led to better GT performance.

\section{GENERAL DISCUSSION}

The present findings replicate our earlier work showing that maintenance of a digit string interferes with both GT

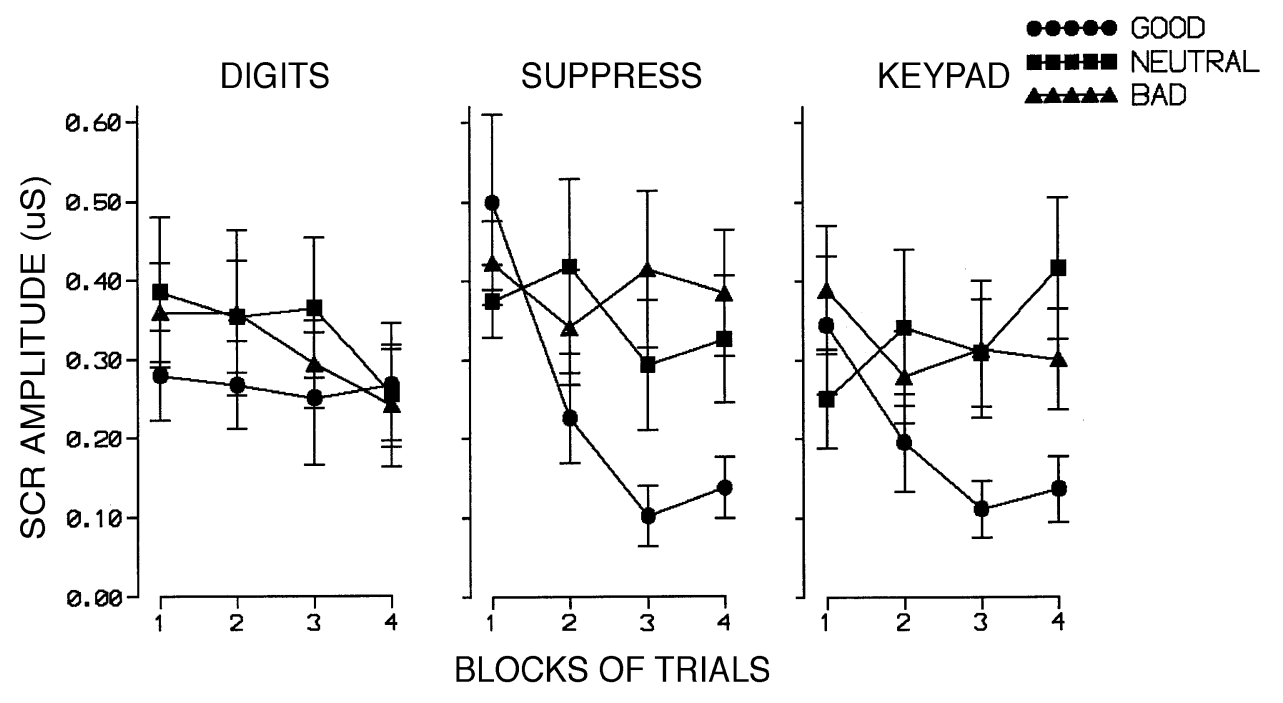

Figure 2. Mean anticipatory skin conductance response (SCR) amplitude during four successive blocks of 25 trials for good (circles), neutral (squares), and bad (triangles) gambling choices. SCR for bad is the mean of SCR for bad1 and bad 2 choices. Error bars are the standard errors of the mean. Performance during the secondary task conditions is shown in the left panel (digit maintenance), the middle panel (articulatory suppression), and the right panel (keypad response). 
choice performance and the development of a somatic marker. The novel result is that articulatory suppression does not interfere with GT performance or the somatic marker. Because articulatory suppression does not disrupt performance, it does not appear that our participants were using a simple verbal mediation strategy to perform the GT. Instead, our present and prior results (Hinson et al., 2002) are best explained by the idea that secondary tasks that interfere with GT performance, such as digit maintenance and random number generation, do so because of their demands on central executive processes. ${ }^{1}$ These data also suggest that central executive resources are required for the development of somatic markers. This is further evidence that poor decision making may occur because problems in WM interfere with the cognitive processes needed to establish anticipatory affective reactions. If VMPFC patients fail to develop somatic markers, even though they have intact WM abilities, the general conclusion that explains the present data and the VMPFC results is that central executive resources are necessary but not sufficient for the development of somatic markers. Refinement of this conclusion awaits more detailed studies of decision-making deficits and executive control.

One way to characterize poor GT performance is that such performance represents an impulsive decision-making style. Impulsive decision making is characteristic not only of VMPFC patients, but also of other groups, including individuals with substance abuse problems (e.g., Bechara et al., 2001; Bechara et al., 2002). We suggest that decisionmaking problems in VMPFC patients and substance abusers may reflect a deeper relationship between impulsiveness and the executive control system. However, impulsiveness covers a wide domain, including motor, motivational, and cognitive components (Evenden, 1999; Patton, Stanford, $\&$ Barratt, 1995). Likewise, there is considerable debate as to what dissociable sets of abilities make up executive control (e.g., Baddeley et al., 2001; Miller \& Cohen, 2001). Given that both impulsiveness and executive control are likely to be multidimensional constructs, we can expect their interrelations to be complex. Some preliminary data in support of this idea were obtained by Whitney, Jameson, and Hinson (2002). We found that risk for substance abuse among college students was related to executive function, but not in terms of a simple linear relationship between WM capacity and risk. Instead, risk for substance abuse was related to an impulsive profile that revealed strengths and weaknesses among different indices of executive function.

Further research into the impulsiveness-executivecontrol relationship promises to yield both theoretical and practical benefits. As we have argued previously (Hinson et al., 2002, 2003), some people who display an impulsive pattern of action may do so because they lack WM resources or other abilities related to executive function needed to assess multiple dimensions of complex decisions. Such people would not make bad decisions because they were unable to delay gratification, which is one interpretation of impulsiveness. Instead, these people would inaccurately evaluate the relative value of short-term and long-term outcomes. If this analysis is correct, we would expect to find that the larger group of people who are generally identified as impulsive, and who are at risk for a variety of real-life problems resulting from poor decision making, can be divided into subsets. One of these subsets would include people with compromise of the executive portions of WM. Other subsets might be poor decision makers for fundamentally different reasons.

\section{REFERENCES}

Baddeley, A. D., Chincotta, D., \& Adlam, A. (2001). Working memory and the control of action: Evidence from task switching. Journal of Experimental Psychology: General, 130, 641-657.

BADDEley, A. D., \& Hitch, G. J. (1974). Working memory. In G. H. Bower (Ed.), The psychology of learning and motivation (Vol. 8, pp. 47-89). New York: Academic Press.

Bechara, A., Damasio, A. R., Damasio, H., \& Anderson, S. W. (1994). Insensitivity to future consequences following damage to human prefrontal cortex. Cognition, 50, 7-15.

Bechara, A., Damasio. H., Tranel, D., \& Damasio, A. R. (1998). Dissociation of working memory from decision-making within the human prefrontal cortex. Journal of Neuroscience, 18, 428-437.

Bechara, A., Damasio, H., \& Damasio, A. R. (2000). Emotion, decision making and the orbitofrontal cortex. Cerebral Cortex, 10, 295-307.

Bechara, A., Dolan, S., Denburg, N., Hindes, A., Anderson, S. W., \& Nathan, P. E. (2001). Decision-making deficits, linked to a dysfunctional ventromedial prefrontal cortex, revealed in alcohol and stimulant abusers. Neuropsychologia, 39, 376-389.

Bechara, A., Dolan, S., \& Hindes, A. (2002). Decision-making and addiction (part II): Myopia for the future or hypersensitivity to reward? Neuropsychologia, 40, 1690-1705.

Bechara, A., Tranel, D., Damasio, H., \& Damasio, A. R. (1996). Failure to respond autonomically to anticipated future outcomes following damage to the prefrontal cortex. Cerebral Cortex, 6, 215-225.

Damasio, A. R. (1994). Descartes' error: Emotion, reason, and the human brain. New York: Grosset/Putnam.

Damasio, A. R. (1998). The somatic marker hypothesis and the possible functions of prefrontal cortex. In A. C. Roberts, T. W. Robbins, \& L. Weiskrantz (Eds.), The prefrontal cortex (pp. 36-50). New York: Oxford University Press.

Dawson, M. E., Schell, A. M., \& Filion, D. L. (2000). The electrodermal system. In J. T. Cacioppo, L. G. Tassinary, \& G. G. Berntson (Eds.), Handbook of psychophysiology (2nd ed., pp. 200-223). New York: Cambridge University Press.

Dunbar, K., \& Sussman, D. (1995). Toward a cognitive account of frontal lobe function: Simulating frontal lobe deficits in normal subjects. In J. Grafman \& K. J. Holyoak (Eds.), Structure and functions of the human prefrontal cortex (pp. 289-304). New York: New York Academy of Sciences.

Evenden, J. L. (1999). Varieties of impulsivity. Psychopharmacology, 146, 348-361.

FUSTER, J. M. (1999). Cognitive functions of the frontal lobes. In B. L. Miller \& J. L. Cummings (Eds.), The human frontal lobes: Functions and disorders (pp. 187-195). New York: Guilford.

Hinson, J. M., Jameson, T. L., \& Whitney, P. (2002). Somatic markers, working memory, and decision making. Cognitive, Affective, \& Behavioral Neuroscience, 2, 341-353.

Hinson, J. M., JAMESON, T. L., \& WhitNey, P. (2003). Impulsive decision making and working memory. Journal of Experimental Psychology: Learning, Memory, \& Cognition, 29, 298-306.

KRAWCZYK, D. C. (2002). Contributions of the prefrontal cortex to the neural basis of human decision making. Neuroscience \& Biobehavioral Reviews, 26, 631-664.

Manes, F., Sahakian, B., Clark, L., Rogers, R., Antoun, N. M., Aitken, M., \& Robbins, T. (2002). Decision making processes following damage to the prefrontal cortex. Brain, 125, 624-639.

Miller, E. K., \& Cohen, J. D. (2001). An integrative theory of prefrontal cortex function. Annual Review of Neuroscience, 24, $167-$ 202. 
Miyake A., \& Shah, P. (1999). Models of working memory. New York: Cambridge University Press.

Patton, J. H., Stanford, M. S., \& Barratt, E. S. (1995). Factor structure of the Barratt Impulsiveness Scale. Journal of Clinical Psychology, 51, 768-774.

SHALLICE, T., \& BURGESS, P. W. (1991). Deficits in strategy application following frontal lobe damage in man. Brain, 114, 727-741.

Smith, E. E., \& Jonides, J. (1999). Storage and executive processes in the frontal lobes. Science, 283, 1657-1661.

Whitney, P., Jameson, T. L., \& Hinson, J. M. (2002, November). Working memory and behavior: From executive control to self-control. Paper presented at the 43rd Annual Meeting of the Psychonomic Society, Kansas City, MO.

\section{NOTE}

1. Secondary tasks used to tax WM may also have effects that are task specific and unrelated to executive functions. For example, digit maintenance could result in poorer GT performance because it disrupts learning of expected values of choice options by means of number-number interference between primary and secondary tasks. To separate the impact of number interference and executive function on GT performance, a future study could employ a letter maintenance, rather than a digit maintenance, secondary task.

(Manuscript received February 19, 2003; revision accepted for publication July 21, 2003.) 